\title{
Correction to: ReMixT: clone-specific genomic structure estimation in cancer
}

\author{
Andrew W. McPherson ${ }^{1,2}$, Andrew Roth ${ }^{3,4}$, Gavin Ha ${ }^{5,6}$, Cedric Chauve ${ }^{7}$, Adi Steif ${ }^{1}$, Camila P. E. de Souza ${ }^{1,2}$,
} Peter Eirew ${ }^{1}$, Alexandre Bouchard-Côté ${ }^{8}$, Sam Aparicio ${ }^{1,2}$, S. Cenk Sahinalp $p^{9,10}$ and Sohrab P. Shah ${ }^{1,2^{*}}$

\section{Correction}

After publication of the original article [1] an error was noted in the Results section (subheading: Probabilistic model) of this manuscript.

The sentence "A mismatch in segment and breakpoint copy number implies that at least one segment end is left disconnected (Fig. $2 \mathrm{~d}$ )."

Should be referring to figure $1 \mathrm{~d}$, thus reading:

"A mismatch in segment and breakpoint copy number implies that at least one segment end is left disconnected (Fig. 1 d)."

These errors were introduced during typesetting, thus the publisher apologizes for this error.

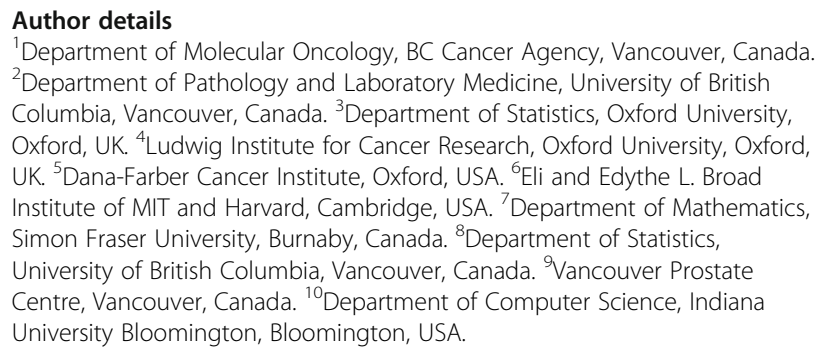

Received: 27 September 2017 Accepted: 27 September 2017

Published online: 06 October 2017

\section{Reference}

1. McPherson AW, Roth A, Ha G, Chauve C, Steif A, de Souza CP, Eirew P, Bouchard-Côté A, Aparicio S, Sahinalp SC, Shah SP. ReMixT: clone-specific genomic structure estimation in cancer. Genome Biol. 2017;18(1):140.

\footnotetext{
* Correspondence: sshah@bccrc.ca

'Department of Molecular Oncology, BC Cancer Agency, Vancouver, Canada ${ }^{2}$ Department of Pathology and Laboratory Medicine, University of British Columbia, Vancouver, Canada

Full list of author information is available at the end of the article
} 